\title{
Severely Constraining Dark-Matter Interpretations of the 21-cm Anomaly
}

\author{
Asher Berlin, ${ }^{1}$ Dan Hooper, ${ }^{2,3,4}$ Gordan Krnjaic, ${ }^{2}$ and Samuel D. McDermott ${ }^{2}$ \\ ${ }^{1}$ SLAC National Accelerator Laboratory, Menlo Park, California 94025, USA \\ ${ }^{2}$ Fermi National Accelerator Laboratory, Theoretical Astrophysics Group, Batavia, Illinois 60510, USA \\ ${ }^{3}$ University of Chicago, Kavli Institute for Cosmological Physics, Chicago, Illinois 60637, USA \\ ${ }^{4}$ University of Chicago, Department of Astronomy and Astrophysics, Chicago, Illinois 60637, USA
}

(Received 9 March 2018; revised manuscript received 19 April 2018; published 2 July 2018)

\begin{abstract}
The EDGES Collaboration has recently reported the detection of a stronger-than-expected absorption feature in the global $21-\mathrm{cm}$ spectrum, centered at a frequency corresponding to a redshift of $z \simeq 17$. This observation has been interpreted as evidence that the gas was cooled during this era as a result of scattering with dark matter. In this Letter, we explore this possibility, applying constraints from the cosmic microwave background, light element abundances, Supernova 1987A, and a variety of laboratory experiments. After taking these constraints into account, we find that the vast majority of the parameter space capable of generating the observed $21-\mathrm{cm}$ signal is ruled out. The only viable models are those in which a small fraction, $\sim 0.3 \%-2 \%$, of the dark matter consists of particles with a mass of $\sim 10-80 \mathrm{MeV}$ and which couple to the photon through a small electric charge, roughly $10^{-6}-10^{-4}$ as large as the electron charge. Furthermore, in order to avoid being overproduced in the early Universe, such models must be supplemented with an additional depletion mechanism, such as annihilations through a $L_{\mu}-L_{\tau}$ gauge boson or annihilations to a pair of rapidly decaying hidden sector scalars.
\end{abstract}

DOI: 10.1103/PhysRevLett.121.011102

Introduction-The Experiment to Detect the Global Epoch of Reionization Signature (EDGES) Collaboration [1] recently reported the measurement of a feature in the absorption profile of the sky-averaged radio spectrum, centered at a frequency of $78 \mathrm{MHz}$ and with an amplitude of $0.5 \mathrm{~K}$. Although such a feature was anticipated to result from the 21-cm transition of atomic hydrogen (at $z \sim 17$ ), the measured amplitude of this signal is significantly larger than expected, at a confidence level of $3.8 \sigma$. If confirmed, this measurement would indicate that either the gas was much colder during the dark ages than expected, or that the temperature of the background radiation was much hotter. It has been argued [2] that standard astrophysical mechanisms [1-7] cannot account for this discrepancy, and that the only plausible explanation for this observation is an interaction between the primordial gas and light dark matter particles, resulting in a significant cooling of the gas $[1,2,4]$ (see also Refs. [8,9]).

In order for dark matter to cool the gas efficiently, it must have specific characteristics. First, equipartition requires that the dark matter particles be fairly light, with masses no larger than a few $\mathrm{GeV}$. Second, if the cross section for dark

Published by the American Physical Society under the terms of the Creative Commons Attribution 4.0 International license. Further distribution of this work must maintain attribution to the author(s) and the published article's title, journal citation, and DOI. Funded by SCOAP ${ }^{3}$. matter scattering with gas is independent of velocity, a variety of constraints, including those from observations of the cosmic microwave background (CMB), would restrict the couplings to well below the values required to explain the observed amplitude of the absorption feature. Velocity-dependent scattering relaxes such constraints, as the average velocities of baryons and dark matter particles were at approximately their minimum value during the cosmic dark ages (due to higher temperatures and structure formation at earlier and later times, respectively). We can maximize the relative impact of dark-matter-baryon scattering during this era by taking $\sigma(v) \propto v^{-4}$. In terms of model building, this consideration favors models in which the dark-matter-baryon interactions are mediated by a particle that is much lighter than the characteristic momentum exchange at $z \sim 17$, with mass $\lesssim \mathrm{keV}$. However, a new particle of this mass would invariably contribute to the energy density of radiation during recombination at a level well above current constraints [10,11], and experimental searches for fifth forces are also very stringent in this mass range $[12,13]$, so a new mediator is ruled out. In light of these considerations, we assume the dark matter carries a small quantity of electric charge (i.e., a millicharge), and thus couples weakly to the photon [14-21].

Model-independent constraints on millicharged dark matter-In Ref. [2], it is claimed that millicharged dark matter particles could cool the gas at $z \sim 17$ to a level consistent with the EDGES measurement $\left(T_{b} \approx 4 \mathrm{~K}\right)$ if the following condition is met [22]: 

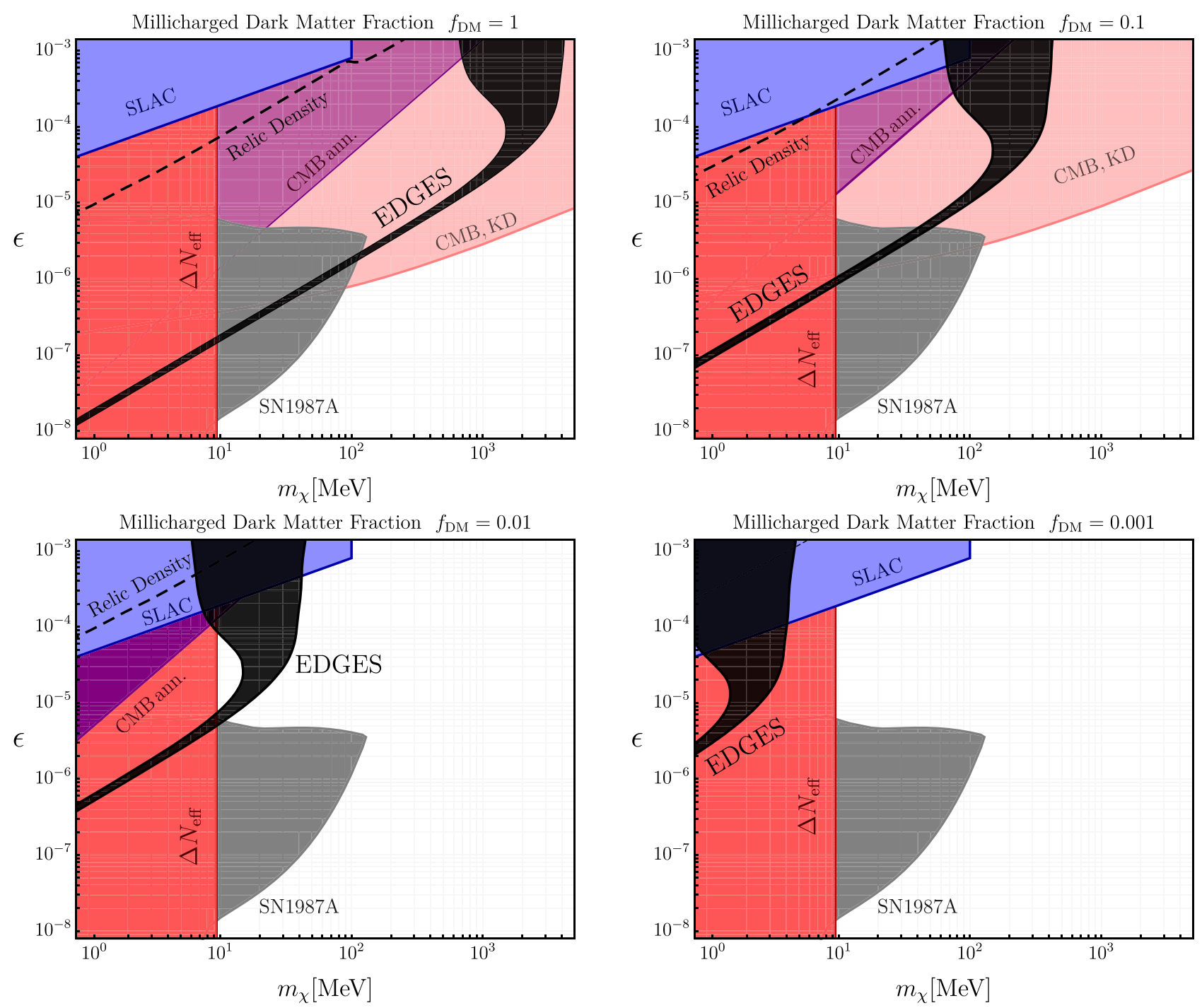

FIG. 1. Constraints on Dirac fermion millicharged dark matter from Supernova 1987A (gray) [23], the SLAC millicharge experiment (blue) [24], the light element abundances produced during big bang nucleosynthesis (red, labeled $\Delta N_{\text {eff }}$ ) [25], and on the impact on the cosmic microwave background of dark matter scattering with baryons (pink, labeled CMB, KD) [14] and dark matter annihilations (purple, labeled CMB ann.) [28]. In each panel, the solid black region can explain the amplitude of the observed 21-cm absorption feature as reported by the EDGES Collaboration [2]. The dashed black line denotes where the thermal relic abundance corresponds to the indicated value of $f_{\mathrm{DM}}$, assuming only millicharge interactions.

$$
\epsilon \approx 1.7 \times 10^{-4}\left(\frac{m_{\chi}}{300 \mathrm{MeV}}\right)\left(\frac{10^{-2}}{f_{\mathrm{DM}}}\right)^{3 / 4},
$$

where $\epsilon \equiv e_{\chi} / e$ is the electric charge of the dark matter, $m_{\chi}$ is the mass of the millicharged dark matter candidate, and $f_{\mathrm{DM}}$ is the fraction of the dark matter that consists of millicharged particles. This expression is valid for $m_{\chi} \lesssim(20-40) \mathrm{MeV} \times\left(f_{\mathrm{DM}} / 10^{-2}\right)$, above which much larger values of $\epsilon$ are required. This expression applies to non-self-conjugate millicharged dark matter.

Millicharged dark matter is subject to a wide range of experimental and astrophysical constraints, some of which we summarize in Fig. 1. We show constraints from observation of Supernova 1987A (gray) [23], from the
SLAC millicharge experiment (blue) [24], and from the light element abundances produced during big bang nucleosynthesis (BBN), assuming entropy transfer to electrons and photons (red) [25], which we label $\Delta N_{\text {eff }}$ (see also Refs. [26,27]). The BBN constraints shift by $\sim O(10 \%)$ if we instead consider entropy transfer to neutrinos. We also show constraints from measurements of the CMB based on dark matter annihilation in the epoch of recombination (purple) [28] (see also Ref. [11]), and on dark matter scattering with baryons (pink) [14]. We have modified the constraint from Ref. [14] by rescaling the limits on $\epsilon$ by a factor of $\sqrt{f_{\mathrm{DM}} \Omega_{\mathrm{DM}} / \Omega_{b}}$ where $\Omega_{i}$ is the fractional energy density in the Universe in species $i$ [29]. Although these results are shown for dark matter in the 
form of a Dirac fermion, most of these constraints would change only very slightly if we were instead to consider a complex scalar. The exception to this is the constraint from dark matter annihilation during recombination [28], which have a $p$-wave suppression of the annihilation cross section for a complex scalar.

Analytical constraints on dark matter scattering with baryons during recombination were presented in Ref. [14] for the case of $f_{\mathrm{DM}}=1$. For $f_{\mathrm{DM}} \gtrsim 0.02$, we rescale these constraints. For smaller values of $f_{\mathrm{DM}}$, one cannot apply this bound because the energy density of this component of the dark matter is smaller than the difference between the (95\% C.L.) upper limit on the baryon density from the CMB [11] and the (95\% C.L.) lower limit on the baryonic density based on BBN [31]. In this regime, the millicharged particles themselves could contribute to the apparent baryonic density as derived from the $\mathrm{CMB}$, evading the CMB constraint even if tightly coupled to the baryon fluid. For this reason, CMB kinetic decoupling bounds do not apply for $f_{\mathrm{DM}} \lesssim 0.02$.

The solid black regions in Fig. 1 represent the parameter space in which the reported amplitude of the $21-\mathrm{cm}$ absorption feature can be explained. For $f_{\mathrm{DM}}=1$, we take this region as presented in Ref. [2]. For $f_{\mathrm{DM}}<1$, we shift these regions in $\epsilon$ and $m_{\chi}$ as found in the numerical solutions presented in Ref. [22] [see Eq. (1)]. The fact that the solid black regions only coincide with the dashed curves in regions that are ruled out by other experiments indicates that the dark matter must be depleted in the early universe by another kind of interaction.

In light of the constraints presented in Fig. 1, we henceforth consider millicharged dark matter in a relatively narrow range of parameter space [32]: $m_{\chi} \sim 10-80 \mathrm{MeV}$, $\epsilon \sim 10^{-6}-10^{-4}$, and with $f_{\mathrm{DM}} \sim 0.003-0.02$.

Depleting the dark matter abundance-A major challenge for millicharged dark matter models which can explain the reported amplitude of the observed 21-cm absorption feature is avoiding overproduction in the early universe. In particular, the $\chi$ equilibrates with the photon bath and acquires a thermal density in the early universe if $\epsilon \gtrsim 10^{-8}\left(m_{\chi} / 10 \mathrm{MeV}\right)^{1 / 2}$. From Eq. (1), this condition is satisfied for all $\epsilon$ that explain the EDGES result, so equilibrium is a generic condition for these models. Once equilibrium is reached, the final relic abundance is determined by the annihilation cross section. Annihilations to charged fermions allow a thermal abundance that makes up the following fraction of the dark matter density: $f_{\mathrm{DM}} \approx 0.04 \times$ $\left(10^{-3} / \epsilon\right)^{2} \times\left(m_{\chi} / 30 \mathrm{MeV}\right)^{2}$ [33]. In Fig. 1, the dashed black lines indicate the regions of parameter space in which the thermal relic abundance from millicharge interactions only corresponds to the quoted value of $f_{\mathrm{DM}}$. The fact that the solid regions do not coincide with the dashed curves in allowed regions of millicharge parameter space indicates that the dark matter must be depleted by other means.
To deplete the thermal abundance of millicharged dark matter to an acceptable level, we consider the possibilities either of supplemental annihilations directly to standard model particles or to new particles within a hidden sector. We require these annihilations either to take place through $p$-wave processes or to result in neutrinos, as measurements of the CMB are stringent for these masses if freeze-out is primarily through $s$-wave annihilation to electromagnetically interacting particles [28].

Annihilation to standard model fermions: For the case of annihilation directly to standard model states, we consider two options for $p$-wave processes: dark matter in the form of a scalar or a fermion which annihilates through a new vector, $V$. In both of these cases, the vector must be heavier than the dark matter itself so that annihilations to standard model fermions dominate over those to $V V$. First, we consider the following interactions which lead to $p$-wave suppressed annihilations:

$$
\begin{aligned}
& \mathcal{L}_{f} \supset V_{\mu}\left(g_{\chi} \bar{\chi} \gamma^{\mu} \gamma^{5} \chi+g_{f} \bar{f} \gamma^{\mu} f\right), \\
& \mathcal{L}_{s} \supset V_{\mu}\left(i g_{\chi} \chi^{*} \partial_{\mu} \chi+g_{f} \bar{f} \gamma^{\mu} f+\text { H.c. }\right),
\end{aligned}
$$

where the dark matter candidate, $\chi$, is a scalar or a fermion, respectively. Although for a Dirac fermion it is also possible to have $p$-wave $\chi \bar{\chi} \rightarrow f \bar{f}$ annihilation through scalar $s$-channel exchange, this possibility is strongly excluded in simple models [34].

In Fig. 2, we plot constraints on models defined as in Eqs. 2(a) and 2(b) in which the millicharged dark matter annihilates through a vector mediator that couples universally to all three species of charged leptons (for details, see Ref. [35]). In this case, constraints from the BABAR [36-38] and E137 [37,39] experiments, along with BBN, exclude the entire range of parameter space that is capable of generating the reported $21-\mathrm{cm}$ signal.

We also consider scenarios in which dark matter annihilates predominantly to neutrinos, with either $s$ - or $p$-wave annihilation:

$$
\begin{aligned}
& \mathcal{L}_{f} \supset V_{\mu}\left(g_{\chi} \bar{\chi} \gamma^{\mu} \chi+g_{\nu} \bar{\nu}_{L} \gamma^{\mu} \nu_{L}\right), \\
& \mathcal{L}_{s} \supset V_{\mu}\left(i g_{\chi} \chi^{*} \partial_{\mu} \chi+g_{\nu} \bar{\nu}_{L} \gamma^{\mu} \nu_{L}+\text { H.c. }\right) .
\end{aligned}
$$

Concretely, we may introduce the anomaly-free gauge group $\mathrm{U}(1)_{L_{\mu}-L_{\tau}}$, leading to couplings to muons, taus, and their respective neutrino species [51-54]. We show in Fig. 2 the parameter space of such a model for a scalar dark matter candidate with interactions as defined in Eq. 3(b). Because this mediator does not directly couple to electrons, the experimental constraints are considerably less restrictive in this case, allowing the dark matter to be sufficiently depleted in the early Universe. This model predicts a contribution to the magnetic moment of the muon, $(g-2)_{\mu}$, that is capable of explaining the measured anomaly [45-48]. It is anticipated 

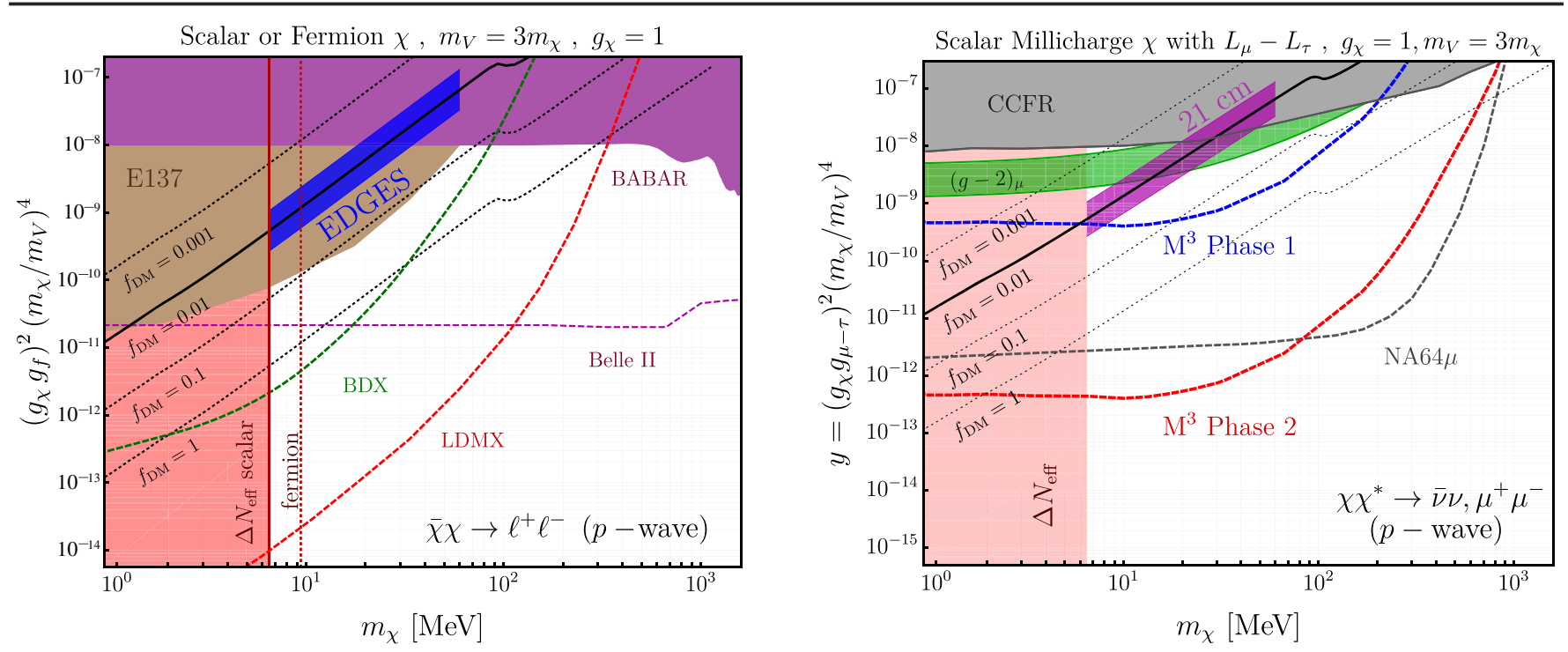

FIG. 2. Constraints on millicharged dark matter, $\chi$, in the viable parameter space of Fig. 1 (bottom left panel) with a new mediator $V$ of mass $m_{V}=3 m_{\chi}$ and gauge coupling $g_{\chi}=1$. (For other choices of this coupling and mass ratio, the constraints are generically stronger.) Left: We assume $V$ couples equally to all three generations of charged standard model leptons. The blue band approximately shows the parameter space which can explain the amplitude of the observed 21-cm absorption feature. This is ruled out by constraints from the $B A B A R$ [36-38] and E137 [37,39] experiments over the entire range of parameter space that is capable of generating the reported signal. Shown as dashed lines are projected constraints from the Belle II [40], BDX [37,41], and LDMX [40,42] experiments. Right: Parameter space in which $V$ couples to $L_{\mu}-L_{\tau}$. Future measurements by the NA64 [43] and $\mathrm{M}^{3}$ experiments [44] are expected to be sensitive to this scenario. In the green band, $V$ resolves the $(g-2)_{\mu}$ anomaly [45-48]. The shaded gray region is constrained by the CCFR experiment $[49,50]$.

that future measurements by the NA64 experiment at the Super Proton Synchrotron (SPS) [43] and the $\mathrm{M}^{3}$ experiment at Fermilab [44] will be sensitive to this scenario.

Annihilation to hidden sector particles: Now we consider the possibility that the dark matter annihilates to unstable hidden sector particles. The $p$-wave requirement implies annihilations to a pair of less massive scalars, $\phi$. For real $\phi$, which leads to the smallest contribution to the energy density of dark radiation, the interaction $\mathcal{L} \supset y \phi \bar{\chi} \chi$ leads to a relic abundance equal to a fraction of the measured dark matter energy density $f_{\mathrm{DM}} \approx 0.008(0.03 / y)^{4}\left(m_{\chi} / 30 \mathrm{MeV}\right)^{2}$ $\left(0.1 / v^{2}\right)$, where $v$ is the relative velocity of the annihilating particles.

The dark matter models under consideration here are strongly constrained by observations of the $\mathrm{CMB}$ and the successful predictions of BBN (see, for example, Refs. [10,55]). If the $\phi$ population is relativistic during BBN, it will contribute $\Delta N_{\text {eff }}=4 / 7$, in excess of the range of values allowed by measurements of the light element abundances [31]. On the other hand, if the $\phi$ population is nonrelativistic and decays after BBN, this will ruin the concordance of the baryon-to-photon ratio as measured during BBN and recombination. In order to evade this constraint, the $\phi$ abundance must be transferred prior to BBN into standard model particles, which then reach equilibrium with both photons and neutrinos, thereby preserving the standard model prediction $N_{\text {eff }}=3.046$ [56].
This could be facilitated, for example, through the rapid decay of the $\phi$. Unless we include additional particle content into our model, the $\phi$ population will decay predominantly to a pair of photons through a $\chi$ loop. We find that there is no combination of parameters that can account for the observed amplitude of the 21-cm absorption feature for which this decay will take place prior to BBN $\left(\tau_{\phi} \lesssim 1 \mathrm{~s}\right)$. Thus, to avoid the stringent constraints from $\mathrm{BBN}$, we must introduce a mechanism to more rapidly deplete the $\phi$ abundance, such as decay to standard model fermions via mixing with the Higgs boson [57]. Such a scenario is bounded from a multitude of other searches [34].

Summary and conclusions.-The recent claim by the EDGES Collaboration of detection of a feature at $78 \mathrm{MHz}$ in the sky-averaged radio spectrum marks a potentially momentous occasion for astrophysics. A signal of the reported magnitude is not compatible with standard astrophysics, potentially pointing to a strong coupling between the baryons and dark matter at a redshift of $z \sim 17$. This striking possibility merits substantial investigation and scrutiny.

In this Letter, we have pointed out several very stringent constraints on a class of dark matter candidates potentially capable of explaining the EDGES 21-cm absorption feature. Since explaining this excess requires a DM-baryon cross section of order $\sigma \sim 10^{-20} \mathrm{~cm}^{2}$, the mediator particle for such an interaction must be lighter than the typical momentum transfer at $z \sim 17$. Since a new mediator with 
couplings of the required size is ruled out by both laboratory and cosmological considerations, we are forced to consider models in which the dark matter possesses a small electric charge. However, such millicharged models are highly constrained by measurements of the cosmic microwave background, light element abundances, and Supernova 1987A. After applying these constraints, we find that the only class of models that could potentially explain the reported $21-\mathrm{cm}$ signal are those in which a small fraction $\sim 0.3 \%-2 \%$ of the dark matter is particles with mass $\sim 10-80 \mathrm{MeV}$ and which couple to the photon through a small electric charge $\epsilon \sim 10^{-6}-10^{-4}$; the remaining dark matter density is comprised of unrelated particles.

Although these features suffice to explain the EDGES excess, the requisite range of masses and couplings problematically overclose the Universe without additional model building: for the parameters of interest, the millicharged particles are in thermal equilibrium with the standard model in the early Universe but their charge is too small to annihilate away its population to an acceptable degree. Additional forces are required to deplete their number density. We have catalogued some simple possibilities for such forces and identified an intriguing scenario in which the depletion occurs through an $L_{\mu}-L_{\tau}$ gauge boson; this can explain the persistent muon $g-2$ anomaly and will be tested by the NA64 and $\mathrm{M}^{3}$ experiments. The parameter space for all such models is fairly narrow and under tension from various laboratory and cosmological constraints.

We would like to thank K. Boddy, C. Dvorkin, R. Essig, V. Gluscevic, W. Hu, M. Kamionkowski, Z. Kang, and J. Muñoz for helpful communication. A. B. is supported by the U.S. Department of Energy under Contract No. DEAC02-76SF00515. This work has been partially supported by Fermi Research Alliance, LLC under Contract No. DE-AC02-07CH11359 with the U.S. Department of Energy, Office of Science, Office of High Energy Physics. The U.S. Government retains and the publisher, by accepting the article for publication, acknowledges that the U.S. Government retains a nonexclusive, paid-up, irrevocable, world-wide license to publish or reproduce the published form of this manuscript, or allow others to do so, for United States Government purposes.

Note added in proof.-Recently, Ref. [61] appeared on a related topic.

[1] J. Bowman, A. Rogers, R. Monsalve, T. Mozdzen, and N. Mahesh, Nature (London) 555, 67 (2018).

[2] R. Barkana, Nature (London) 555, 71 (2018).

[3] A. Cohen, A. Fialkov, R. Barkana, and M. Lotem, Mon. Not. R. Astron. Soc. 472, 1915 (2017).

[4] A. Fialkov, R. Barkana, and A. Cohen, preceding Letter, Phys. Rev. Lett. 121, 011101 (2018).
[5] C. Feng and G. Holder, arXiv:1802.07432.

[6] A. Ewall-Wice, T. C. Chang, J. Lazio, O. Dore, M. Seiffert, and R. A. Monsalve, arXiv:1803.01815.

[7] J. Mirocha, G. J. A. Harker, and J. O. Burns, Astrophys. J. 813, 11 (2015).

[8] H. Tashiro, K. Kadota, and J. Silk, Phys. Rev. D 90, 083522 (2014).

[9] J. B. Muñoz, E. D. Kovetz, and Y. Ali-Haïmoud, Phys. Rev. D 92, 083528 (2015).

[10] H. Vogel and J. Redondo, J. Cosmol. Astropart. Phys. 02 (2014) 029.

[11] P. A. R. Ade et al. (Planck Collaboration), Astron. Astrophys. 594, A13 (2016).

[12] E. G. Adelberger, B. R. Heckel, S. A. Hoedl, C. D. Hoyle, D. J. Kapner, and A. Upadhye, Phys. Rev. Lett. 98, 131104 (2007).

[13] D. J. Kapner, T. S. Cook, E. G. Adelberger, J. H. Gundlach, B. R. Heckel, C. D. Hoyle, and H. E. Swanson, Phys. Rev. Lett. 98, 021101 (2007).

[14] S. D. McDermott, H.-B. Yu, and K. M. Zurek, Phys. Rev. D 83, 063509 (2011).

[15] S. Davidson, S. Hannestad, and G. Raffelt, J. High Energy Phys. 05 (2000) 003.

[16] L. Chuzhoy and E. W. Kolb, J. Cosmol. Astropart. Phys. 07 (2009) 014

[17] C. Dvorkin, K. Blum, and M. Kamionkowski, Phys. Rev. D 89, 023519 (2014).

[18] A. D. Dolgov, S. L. Dubovsky, G. I. Rubtsov, and I. I. Tkachev, Phys. Rev. D 88, 117701 (2013).

[19] S. L. Dubovsky, D. S. Gorbunov, and G. I. Rubtsov, Pis'ma Zh. Eksp. Teor. Fiz. 79, 3 (2004); [JETP Lett. 79, 1 (2004)].

[20] D. Feldman, Z. Liu, and P. Nath, Phys. Rev. D 75, 115001 (2007).

[21] New mediator degree(s) of freedom are disfavored by measurements sensitive to the Hubble rate at early times, as discussed in more detail below. Furthermore, a scalar with Higgs mixing would be suppressed by the standard model Yukawa couplings, which are all small; and a scattering cross section that scales like $v^{-2}$, as would occur for a dark matter particle with a dipole moment, is more constrained by considerations from other epochs because it is comparatively less enhanced during dark ages.

[22] J. B. Muñoz and A. Loeb, arXiv:1802.10094.

[23] J. H. Chang, R. Essig, and S. D. McDermott, arXiv:1803 .00993.

[24] A. A. Prinz et al., Phys. Rev. Lett. 81, 1175 (1998).

[25] C. Boehm, M. J. Dolan, and C. McCabe, J. Cosmol. Astropart. Phys. 08 (2013) 041.

[26] K. M. Nollett and G. Steigman, Phys. Rev. D 91, 083505 (2015).

[27] G. Steigman and K. M. Nollett, Phys. Procedia 61, 179 (2015).

[28] T. R. Slatyer, Phys. Rev. D 93, 023527 (2016).

[29] Reference [30] has recently presented an even more stringent constraint on low-velocity dark matter-baryon scattering.

[30] W. L. Xu, C. Dvorkin, and A. Chael, arXiv:1802.06788.

[31] R. H. Cyburt, B. D. Fields, K. A. Olive, and T.-H. Yeh, Rev. Mod. Phys. 88, 015004 (2016). 
[32] Because of the unavoidable addition of extra degrees of freedom at $\mathrm{CMB}$ and $\mathrm{BBN}$ times, we will not assume that the millicharge is accompanied by an extremely light dark photon [10].

[33] G. Steigman, B. Dasgupta, and J. F. Beacom, Phys. Rev. D 86, 023506 (2012).

[34] G. Krnjaic, Phys. Rev. D 94, 073009 (2016).

[35] E. Izaguirre, G. Krnjaic, P. Schuster, and N. Toro, Phys. Rev. Lett. 115, 251301 (2015).

[36] J. P. Lees et al. (BABAR Collaboration), Phys. Rev. Lett. 119, 131804 (2017).

[37] E. Izaguirre, G. Krnjaic, P. Schuster, and N. Toro, Phys. Rev. D 88, 114015 (2013).

[38] R. Essig, J. Mardon, M. Papucci, T. Volansky, and Y.-M. Zhong, J. High Energy Phys. 11 (2013) 167.

[39] J. D. Bjorken, S. Ecklund, W. R. Nelson, A. Abashian, C. Church, B. Lu, L. W. Mo, T. A. Nunamaker, and P. Rassmann, Phys. Rev. D 38, 3375 (1988).

[40] E. Izaguirre, G. Krnjaic, P. Schuster, and N. Toro, Phys. Rev. D 91, 094026 (2015).

[41] M. Battaglieri et al. (BDX Collaboration), arXiv:1607 .01390 .

[42] M. Battaglieri et al., arXiv:1707.04591.

[43] S. N. Gninenko, N. V. Krasnikov, and V. A. Matveev, Phys. Rev. D 91, 095015 (2015).

[44] Y. Kahn, G. Krnjaic, N. Tran, and A. Whitbeck, arXiv: 1804.03144.

[45] G. W. Bennett et al. (Muon g-2 Collaboration), Phys. Rev. D 73, 072003 (2006).

[46] K. Hagiwara, R. Liao, A. D. Martin, D. Nomura, and T. Teubner, J. Phys. G 38, 085003 (2011).
[47] M. Davier, A. Hoecker, B. Malaescu, and Z. Zhang, Eur. Phys. J. C 71, 1515 (2011); [72, 1874(E) (2012)].

[48] F. Jegerlehner and A. Nyffeler, Phys. Rep. 477, 1 (2009).

[49] W. Altmannshofer, S. Gori, M. Pospelov, and I. Yavin, Phys. Rev. Lett. 113, 091801 (2014).

[50] S. R. Mishra et al. (CCFR), Phys. Rev. Lett. 66, 3117 (1991).

[51] X. G. He, G. C. Joshi, H. Lew, and R. R. Volkas, Phys. Rev. D 43, R22 (1991).

[52] X.-G. He, G. C. Joshi, H. Lew, and R. R. Volkas, Phys. Rev. D 44, 2118 (1991).

[53] S. Baek, N. G. Deshpande, X. G. He, and P. Ko, Phys. Rev. D 64, 055006 (2001).

[54] M. Pospelov, Phys. Rev. D 80, 095002 (2009).

[55] C. Brust, D. E. Kaplan, and M. T. Walters, J. High Energy Phys. 12 (2013) 058.

[56] G. Mangano, G. Miele, S. Pastor, and M. Peloso, Phys. Lett. B 534, 8 (2002).

[57] Alternatively, the $\phi$ abundance could be depleted by efficient 3-to-2 processes [58-60], though the details of such an implementation are nontrivial.

[58] E. D. Carlson, M. E. Machacek, and L. J. Hall, Astrophys. J. 398, 43 (1992).

[59] Y. Hochberg, E. Kuflik, H. Murayama, T. Volansky, and J. G. Wacker, Phys. Rev. Lett. 115, 021301 (2015).

[60] D. Pappadopulo, J. T. Ruderman, and G. Trevisan, Phys. Rev. D 94, 035005 (2016).

[61] G. D'Amico, P. Panci, A. Strumia, following Letter, Phys. Rev. Lett. 121, 011103 (2018). 